\title{
Evidence for angiotensin II type 2 receptor-mediated cardiac myocyte enlargement during in vivo pressure overload
}

\author{
Takaaki Senbonmatsu, ${ }^{1}$ Sahoko Ichihara, ${ }^{1}$ Edward Price, Jr., ${ }^{1}$ F. Andrew Gaffney, ${ }^{2}$ \\ and Tadashi Inagami ${ }^{1}$
}

${ }^{1}$ Department of Biochemistry, Vanderbilt University School of Medicine, Nashville, Tennessee, USA
${ }^{2}$ Department of Medicine, Vanderbilt University School of Medicine, Nashville, Tennessee, USA
Address correspondence to: Tadashi Inagami, Department of Biochemistry, Vanderbilt University School of Medicine,
Nashville, Tennessee 37232-0146, USA. Phone: (615) 322-4347; Fax: (615) 322-3201; E-mail: tadashi.inagami@vanderbilt.edu.

Received for publication April 6, 2000, and accepted in revised form June 13, 2000.

The pathophysiological roles of the angiotensin II type 2 receptor $\left(\mathrm{AT}_{2}\right)$ in cardiac hypertrophy remain unclear. By the targeted deletion of mouse $A T_{2}$ we were able to prevent the left ventricular hypertrophy resulting from pressure overload, while cardiac contractile functions remained normal. This implies that $\mathrm{AT}_{2}$ is a mediator of cardiac hypertrophy in response to increased blood pressure. The effects of $A T_{2}$ deletion were independent of activation of embryonic genes for cardiac hypertrophy. However, $\mathrm{p} 70^{\mathrm{s} 6 \mathrm{k}}$, one of the key factors in cardiac hypertrophy, was markedly and specifically reduced in the ventricles of Agtr2-/Y mice. We propose that $\mathrm{p}^{-} 0^{\mathrm{S} 6 \mathrm{k}}$ plays a major role in $\mathrm{AT}_{2}$-mediated ventricular hypertrophy.

This article may have been published online in advance of the print edition. The date of publication is available from the JCI website, http://www.jci.org. J. Clin. Invest. 106:R25-R29 (2000).

\section{Introduction}

Cardiac hypertrophy is an adaptive response of the heart to several forms of cardiac disease. Although sustained hypertrophy is an initial compensatory mechanism to preserve cardiac function, it is also a major risk factor for congestive heart failure and sudden death.

Angiotensin II (Ang II) plays an important role in the development of cardiac hypertrophy (1). It is supplied by the circulating renin-angiotensinogen system and also is generated locally (2). Treatment with angiotensin-converting enzyme inhibitors or Ang II-receptor antagonists induces marked but incomplete regression and prevention of the cardiac hypertrophy (3).

Of two major subtypes of Ang II receptors, type $1\left(\mathrm{AT}_{1}\right)$ and type 2 $\left(\mathrm{AT}_{2}\right)$, only the latter appears to increase under pathological conditions including cardiac hypertrophy (4). However, its function remains controversial. Recent studies reported that pressure overload-induced left ventricular hypertrophy ( $\mathrm{LVH})$ still occurs in mice lacking the $A T_{1 A}$ gene
$\left(A g t r 1 A^{-/-}\right)(5,6)$. In vitro studies on $\mathrm{AT}_{2}$ in cultured cells have been difficult because its functions are often poorly reproducible. In the present in vivo studies, $A T_{2}$ gene-targeted mice $\left(A g t r 2^{-} / Y\right)$ were used to delineate the role of $\mathrm{AT}_{2}$ in cardiac hypertrophy. The well-established ability of Ang II to induce cardiac hypertrophy prompted us to investigate the hypothesis that pressure overload-induced LVH would be prevented in the Agtr2-/Y mice.

\section{Methods}

Agtr2- $/ Y$ mice. Ten-week-old male Agtr2- $/ Y(n=9)$ and wild-type $(n=7)$ mice weighing $22-24 \mathrm{~g}$ were used. The Agtr2-/Y mice were produced as described previously (7), by using E 141 embryonic stem cells from male 129 Ola mice and blastocysts derived from the C57BL/ 6 strain (The Jackson Laboratory, Bar Harbor, Maine, USA), backcrossed to C57BL/ 6 genetic background by mating C57BL/ 6 males to heterozygous $\mathrm{AT}_{2}$ females. The Agtr2-/Y mice from the 8th generation of the backcross to C57BL/ 6 mice were used. The wild-type mice were generat- ed as littermates by the same mating procedure. Experimental protocols were approved by Vanderbilt Animal Care Committee.

Pressure overload by aortic constriction. Pressure overload was produced by a modification of the published banding method of the abdominal aorta (8). As controls, sham-operated mice were produced in both the Agtr2-/Y and wild-type groups, using a procedure that was identical except for the aortic banding above the left renal artery.

Blood pressure measurement. To ascertain the pressure gradient of aortic constriction, the left carotid and femoral arteries were cannulated with stretched PE 10 intramedic polyethylene tubing (Clay Adams, division of Becton Dickinson and Co., Parsippany, New Jersey, USA) after characterization of cardiac properties. Blood pressure was measured under anesthesia with ketamine $(50 \mathrm{mg} / \mathrm{kg})$ and xylazine $(20 \mathrm{mg} / \mathrm{kg})$.

Echocardiography. Transthoracic echocardiography was performed with a Sonos 5500 with an S12 transducer (12 MHz) (Hewlett-Packard, Andover, Massachusetts, USA). Mice were weighed, anesthetized with ketamine $(50 \mathrm{mg} / \mathrm{kg})$ and xylazine $(20 \mathrm{mg} / \mathrm{kg})$, and settled in the left decubitus or supine position (9). Good two-dimensional views of the left ventricle were obtained for guided M-mode measurements of the intraventricular septum (IVS), left ventricular end-diastolic diameter (LVDd), left ventricular posterior wall (LVPW), and left ventricular end-systolic diameter (LVDs). Percent fractional shortening (\%FS) was calculated by the formula: [(LVDd 
- LVDs)/LVDd] $\times 100$. Mean of three measurements was determined for each mouse. The echocardiographic left ventricular mass (LVM) was calculated using the specific gravity of myocardium $\left(1.055 \mathrm{mg} / \mathrm{mm}^{3}\right)$ and the cube formula: $\mathrm{LVM}=1.055 \times[(\mathrm{IVS}+$ LVPW + LVDd $)^{3}-\left(\right.$ LVDd $\left.^{3}\right]$ (ref. 10). After the echocardiographic and pressure gradient study, mice were euthanized. Major blood vessels, both atria, and the right ventricle were removed from the heart. Then the left ventricle was dissected and weighed. This value was used as the autopsied left ventricular weight.

Histology. Histological analysis was performed on aortic-banded Agtr2-/Y and wild-type mice as well as shamoperated Agtr2-/Y and wild-type mice. Briefly, hearts were isolated after perfusion with $40 \mathrm{mM} \mathrm{KCl}$, fixed in $4 \%$ paraformaldehyde with PBS, dehydrated in graded ethanol solution, and transferred to xylene and then into paraffin. The paraffin-embedded hearts were sectioned at $4 \mu \mathrm{m}$ and subsequently were stained with hematoxylin, eosin, and Van Gieson solution. Left ventricular myocyte crosssectional area (CSA) was measured on sections of mid-free wall of the left ventricle. Suitable cross sections were defined as having nearly circular capillary profiles and circular to oval myocyte cross sections. The outer borders of the myocytes were traced and myocyte areas were calculated with an NIH image system (NIH, Research Service Branch). Approximately 100 cells were counted per sample and the average was used for analysis.

Western blot analysis. Western blot analysis was performed for ventricular extracts of aortic-banded Agtr2-/Y and wild-type mice and sham-operated Agtr2- $/ Y$ and wild-type mice as previously reported (11). Rabbit polyclonal phosphospecific antibodies to $\mathrm{p} 70^{\text {s6k }}$, extracellular signal-regulated kinase (ERK), p38 mitogen-activated protein kinase (p38 MAPK), and Jun $\mathrm{NH}_{2}$-terminal kinase (JNK) (New England Biolabs Inc., Beverly, Massachusetts, USA) and rabbit polyclonal antibodies to p $70^{\mathrm{S} 6 \mathrm{k}}$ (New England Biolabs Inc.), collagen type I (Calbiochem-Novabiochem Corp., La Jolla, California, USA), and calcineurin (Santa Cruz Biotechnology Inc., Beverly, Massachusetts, USA) were used. ECL (Amersham Pharmacia Biotech, Piscataway, New Jersey, USA) was used for detection of immunoreactive bands. The outer borders of the bands were traced and the areas and densities were determined with the NIH image system.

Northern blot analysis. Northern blot analysis of Nkx 2.5, GATA4, myocyte enhancer factor 2C (MEF2C), $\beta$-myosin heavy chain ( $\beta$-MHC), atrial natriuretic peptide (ANP), and GAPDH were performed as previously reported (12).

$R$ Nase protection assay and calcineurin activity. Mouse $\mathrm{AT}_{1}$ RNase protection assay was performed on aorticbanded Agtr2-/Y and wild-type mice as previously reported (13). The following primers for PCR were used: sense 5'-GGTGGGAATATTTGGAAACAG-3' and antisense 5'-AAGAAGAAAAGCACAATCGCC-3'.

Calcineurin enzyme activity was determined as previously reported (14).

Statistical analysis. For all statistical tests, multiple comparisons were performed by two-way ANOVA and Fisher's exact probability test. A $P$ value less than 0.05 was considered significant.

\section{Results}

Effect of the pressure overload on systolic pressure. Changes in the left carotid artery systolic pressure after aortic constriction were determined by the direct method. Ten weeks after abdominal aortic constriction, left carotid artery systolic pressure increased significantly as compared with sham-operated mice. The magnitude of increase was similar in aortic-banded Agtr2-/Y (56 \pm $6 \mathrm{mmHg}$ ) and wild-type mice (62 \pm 7 $\mathrm{mmHg}$ ) (Figure 1a).

Determination of effects of the pressure overload on left ventricular dimension and function by echocardiography. In the native state, IVSs and LVPWs of the $\operatorname{Agtr}^{-} / \mathrm{Y}$ mice were thinner than those of the wild-type mice. Surprisingly, in the Agtr2-/Y mice aortic constriction with concomitant elevation in systolic blood pressure induced little or no thickening of the ventricular walls, whereas marked LVH was seen in aortic-banded wild-type mice (Figure 1b). LVM in the Agtr2-/Y mice remained at almost the same levels as in sham-operated animals, whereas in the wild-type, a marked
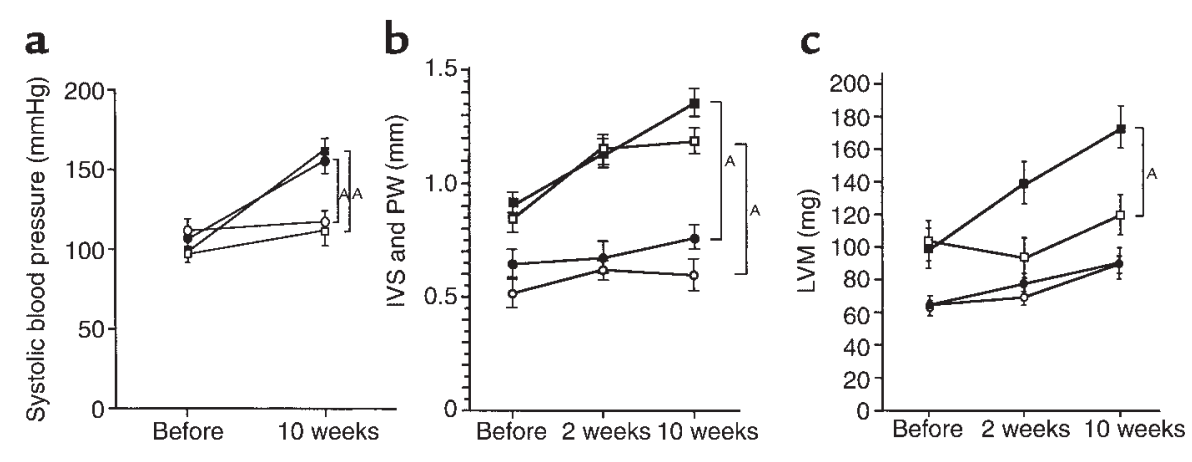

\section{Figure 1}

(a) Systolic blood pressure in Agtr2-/Y and wild-type mice. Filled circles: aortic-banded Agtr2-/Y mice; filled squares: aortic-banded wild-type mice; open circles: sham-operated Agtr2-/Y mice; open squares: sham-operated wild-type mice. ${ }^{A} P<0.05$. (b) IVS and LVPW in aortic-banded Agtr2-/Y and wild-type mice. Filled circles: IVS in aortic-banded Agtr2-/Y mice; filled squares: IVS in aortic-banded wild-type mice; open circles: LVPW in aortic-banded Agtr2-/Y mice; open squares: LVPW in aortic-banded wild-type mice. ${ }^{A} P<0.05$. (c) LVM of Agtr2-/Y and wildtype mice. Filled circles: aortic-banded Agtr2- $/ Y$ mice; open circles: sham-operated Agtr2- $/ Y$ mice; filled squares: aortic-banded wild-type mice; open squares: sham-operated wild-type mice. ${ }^{A} P<0.05$. 

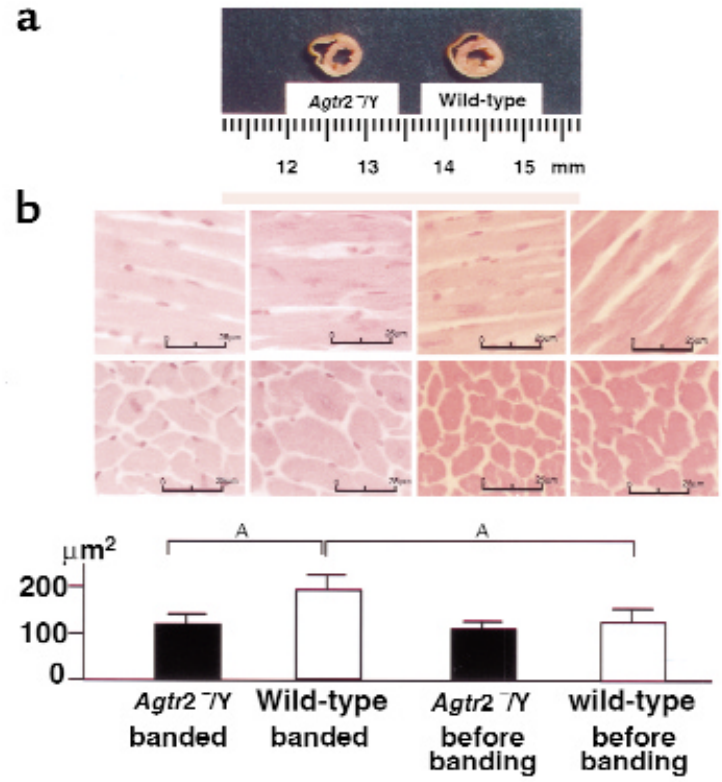
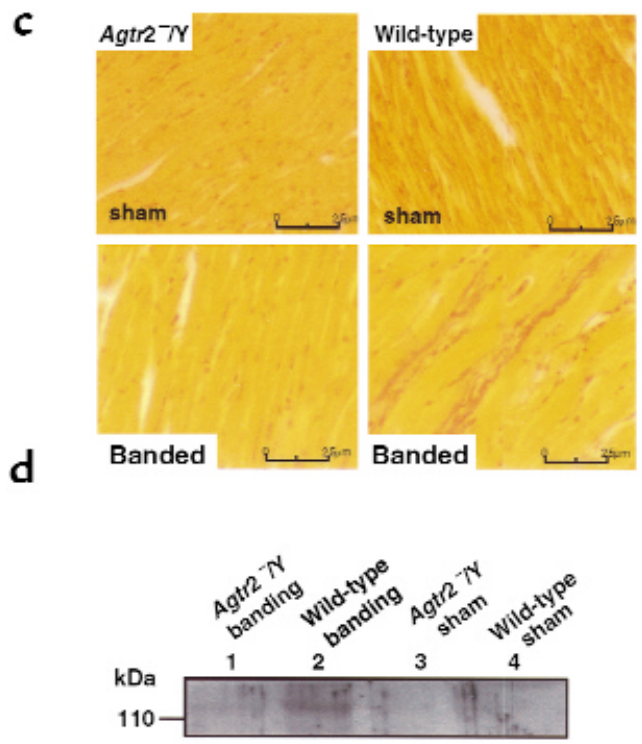

\section{Figure 2}

Pathological analysis in the Agtr2-/Y and wild-type mouse heart. (a) Cross section of midportion of left ventricle in aortic-banded Agtr2-/Y and wild-type mice. (b) Left ventricular CSA in aortic-banded Agtr2- $/ Y$ and wild-type mice, and in Agtr2-/Y and wild-type mice before banding. ${ }^{A} P<0.05$. (c and d) Collagen type I in aortic-banded Agtr2- $/ Y$ and wild-type mouse hearts and in sham-operated Agtr2- $/ Y$ and wild-type mouse hearts. Lane 1: aortic-banded Agtr2-/Y; lane 2: aortic-banded wild-type; lane 3: sham-operated Agtr2-/Y; lane 4: sham-operated wildtype mouse hearts. Data are representative of three independent experiments with nearly identical results.

difference was seen between the sham and aortic-constricted groups (Figures $1 \mathrm{c}$ and $2 \mathrm{a}$ ). In contrast with IVS, LVPW, and LVM, we did not see significantly different CSAs between the Agtr2-/Y and wild-type before bonding. CSAs in aortic-banded Agtr2-/Y mice increased slightly as compared with those in sham-operated Agtr2-/Y mice. However, they were considerably less than those in aortic-banded wild-type mice (Figure $2 b)$. The correlation of autopsied left ventricular weight and LVM was excellent, with correlation constants of 0.70 and 0.85 , respectively (data not shown).

Left ventricular functions of Agtr2- $/ Y$ and wild-type mice. To determine whether the unique morphological features of the left ventricles and the absence of hypertrophic response to aortic constriction in Agtr2-/Y mice are related to any functional abnormality, we determined LVDd and \%FS. Despite thinner left ventricular wall thickness and a markedly reduced hypertrophic response to pressure overload, these indices were comparable between the Agtr2-/Y and wild-type mice (Figure 3, a and b). $\mathrm{AT}_{1}$ expres- sion examined by RNase protection assay was also comparable between the Agtr2 $^{-} / \mathrm{Y}$ and wild-type mice, and it remained comparable between the two groups 10 weeks after aortic constriction (data not shown). Thus, the features of the hearts of the Agtr2-/Y mice are (a) normal ventricular function, (b) thinner ventricular wall thickness, and, most interestingly, (c) a markedly suppressed hypertrophic response to pressure overload.

Reduced collagen type I in Agtr2- /Y mice. Left ventricular and interstitial collagen type I was markedly reduced in aortic-banded Agtr2-/Y mice compared with aortic-banded wild-type mice (Figure 2, c and d).

Markedly reduced $p 70^{S 6 k}$ in Agtr2- $/ Y$ mice. To determine the signaling mechanisms underlying the resistance of the left ventricle of the Agtr2-/Y mouse to growth stimulation by pressure overload, we compared the expression of various compounds involved in ventricular growth stimulation between the Agtr2- $/$ Y and wild-type mice. $\mathrm{p} 70^{\text {s6k }}$ is one of the key factors in cardiac hypertrophy (15). We found that phosphorylated $\mathrm{p}^{7} 0^{\mathrm{S} 6 \mathrm{k}}$ and total $\mathrm{p} 70^{\mathrm{S} 6 \mathrm{k}}$ were markedly reduced in the Agtr2 $/ Y$ mice compared with the wild-type mice. Although aortic constriction increased both, their levels were still markedly below those of similarly treated wild-type mice (Figure 4a). These observations suggest that the markedly attenuated hypertrophic response of the Agtr2-/Y mouse may be due, in large part, to suppression of p $70^{56 \mathrm{k}}$ expression and activation in mice lacking the $\mathrm{AT}_{2}$ receptor.

On the other hand, no difference was observed between the Agtr2-/Y and wild-type mice in the following substances, which are considered to stimulate hypertrophy: phosphorylated ERK, p38 MAPK, JNK, calcineurin and calcineurin enzyme activity. Aortic banding did increase these factors slightly but to similar extents in the Agtr2-/Y and wild-type mice (data not shown). No difference was detected in mRNA of the following well-known markers of ventricular hypertrophy: Nkx 2.5, GATA4, MEF2C, $\beta$-MHC, and ANP. In the native state, there was no significant difference between the groups in any of these markers. Two days after surgery, they were increased equally in both groups and reduced to base lines at 2 weeks after surgery (Fig- 

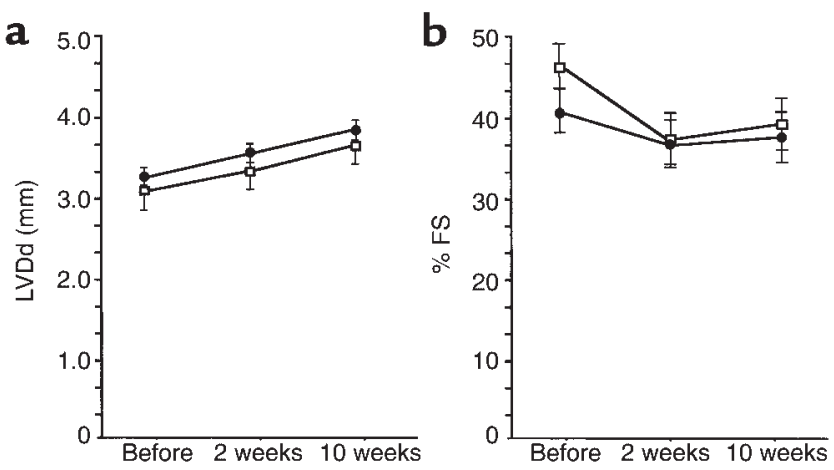

\section{Figure 3}

Heart function in aortic-banded Agtr2- $/ Y$ and wild-type mice. (a) LVDd in aortic-banded Agtr2- $/ Y$ (filled circles) and wild-type (open squares) mice. (b) \%FS in aortic-banded Agtr2- $Y$ (filled circles) and wild-type (open squares) mice. ure $4 \mathrm{~b})$. Based on these results, we submit that attenuation of the signal pathway linking $\mathrm{AT}_{2}$ to $\mathrm{p} 70^{\mathrm{S} 6 \mathrm{k}}$ activation may be involved in the resistance to pressure overload-induced LVH in Agtr2-/Y mice.

\section{Discussion}

The present studies demonstrate that pressure overload failed to induce $\mathrm{LVH}$ in the Agtr2-/Y mice, whereas substantial LVH occurred in similarly treated wild-type mice. We also found that $\mathrm{p} 70^{\mathrm{S} 6 \mathrm{k}}$ was markedly and specifically reduced whereas other hypertrophystimulating genes were not reduced.

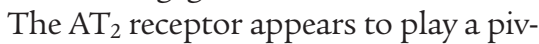
otal role in regulating pressure overload-induced LVH. Although many LVH-inducing genes and proteins have been described, by gain-of-function studies (16-18), only a few have been shown to be responsible for cardiac hypertrophy by loss-of-function studies (19). Particularly, we are not aware of any previous reports on the prevention of pressure overload-induced cardiac hypertrophy by deletion of the $\mathrm{AT}_{2}$ receptor. The present results indicate a role for the expressed $\mathrm{AT}_{2}$ receptor in the development of LVH in response to pressure overload.

Several recent reports show that the $\mathrm{AT}_{2}$ receptor functions counteract those of the $\mathrm{AT}_{1}$ receptor (20). In the present studies, we utilized the Agtr2-/Y mice to delineate the mechanism of hypertrophy suppression associated with the chronic effect of the absence of the $\mathrm{AT}_{2}$ receptor. In their native states, the Agtr2- $/ Y$ mice had thinner ventricular walls than those of the wild-type, as shown in Figure 1, b and c. However, their cardiac function was normal. After chronic aortic constriction, the cardiac function remained normal and cardiac enlargement was not seen in the Agtr2- $/ Y$ mice. This indicates that the Agtr2-/Y mice are not a model of dilated cardiomyopathy.

The ability to maintain normal cardiac function under pressure overload without evoking $\mathrm{LVH}$ is remarkable and seems to imply a primary pathological role of the LVH itself, rather than a secondary effect to compensate the pressure overload (21). It is surprising that the left ventricular myocardium was able to maintain normal fiber length and contractility with no evidence of left ventricular dilation and failure. The markedly reduced collagen type I in aortic-banded Agtr2-/Y mice may explain at least partly the normal contractile function $(22,23)$.

Since cardiac hypertrophy is dependent mainly on protein synthesis, and the 56 kinases, particularly $\mathrm{p} 70^{\mathrm{S} 6 \mathrm{k}}$, play a pivotal regulatory role, the present observations suggest that the marked

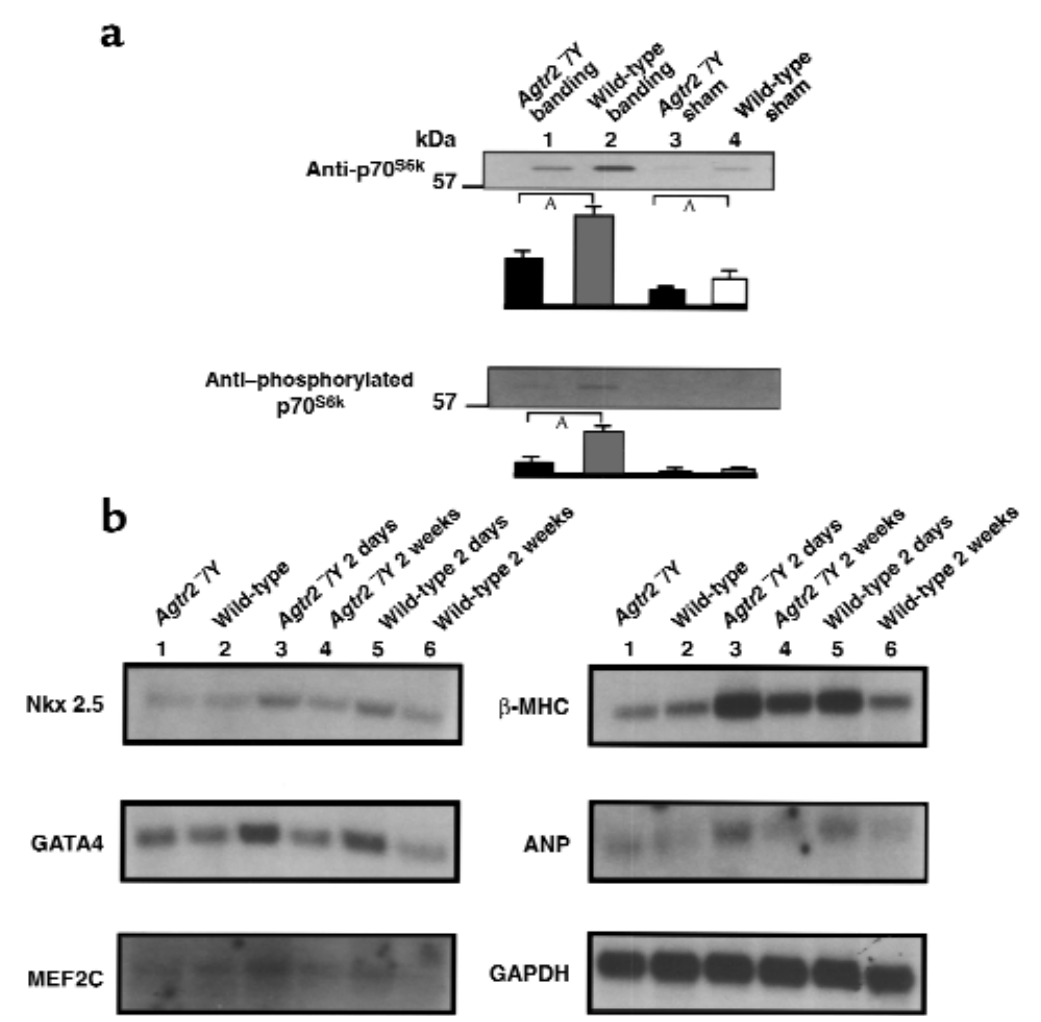

Figure 4

(a) Phosphorylated $p 70^{56 \mathrm{k}}$ and total $\mathrm{p} 70^{\mathrm{s} 6 \mathrm{k}}$ in the Agtr2- $\mathrm{Y}$ and wild-type mouse hearts. Lane 1: aortic-banded Agtr2-/Y; lane 2: aortic-banded wild-type; lane 3: sham-operated Agtr2-/Y; lane 4: sham-operated wild-type. ${ }^{A} P<0.05$. Data are representative of three independent experiments with nearly identical results. (b) Nkx 2.5, GATA4, MEF2C, $\beta$-MHC, ANP, and GAPDH in aortic-banded Agtr2- $/ Y$ and wild-type mouse hearts. Lane 1: Agtr2 $/ Y$ before surgery; lane 2: wild-type before surgery; lane 3: Agtr2-/Y 2 days after surgery; lane 4: Agtr2-/Y 2 weeks after surgery; lane 5: wild-type 2 days after surgery; lane 6: wild-type 2 weeks after surgery. Data are representative of three independent experiments with nearly identical results. 
resistance to hypertrophy in the Agtr2-/Y mice implicates suppression of $\mathrm{p} 70^{\mathrm{S} 6 \mathrm{k}}$. The marked reduction in the myofilament diameter in the Agtr2-/Y mice can also be explained by the reduced $\mathrm{p} 70^{\mathrm{S} 6 \mathrm{k}}(15)$. Thus, the present findings suggest the possibility that induction of $\mathrm{LVH}$ requires the expression of $\mathrm{AT}_{2}$, and the regulation of LVH by $\mathrm{AT}_{2}$ may use a pathway distinct from that of $\mathrm{AT}_{1}$, even though both may converge to activate $\mathrm{p} 70^{\mathrm{S} 6 \mathrm{k}}(24)$. These observations suggest that longterm pressure overload effect via $\mathrm{AT}_{2}$ regulates the expression of $\mathrm{p}^{76 \mathrm{~s}}$, which may regulate ventricular myocyte protein synthesis.

Several MAPKs, particularly ERKs 1 and 2 , are considered to be key factors regulating cell growth (25). However, no difference was seen in the phosphorylated forms of any of the three MAPKs between the Agtr2-/Y and wild-type mice. Since expression of ANP in aorticbanded Agtr2-/Y mice, a sensitive marker for ventricular response to pressure overload (26), is not different from that of aortic-banded wild-type mice, transcriptional mechanisms related to ANP gene expression may not be involved in the $\mathrm{AT}_{2}$-related hypertrophy. Recently, mechanisms involving calcineurin and nuclear factor of activated $\mathrm{T}$ cells -3 (NFAT-3), and/or activation of MEF2C by $\mathrm{Ca}^{2+}$-calmodulin-activated protein kinases II and IV, have been proposed for cardiac hypertrophy (27). Again, these factors do not appear to be involved in the resistance to $\mathrm{LVH}$ in Agtr2-/Y mice. In the Agtr2-/Y mice, all of the hypertrophy-inducing factors discussed above were comparable to those of the wild-type mice.

The almost complete abrogation of hypertrophic response in the Agtr2-/Y mouse heart may indicate that the presence of $\mathrm{AT}_{2}$ is a major factor in the cardiac hypertrophy. This hypothesis finds support in the observation of Harada et al. (5) and Hamawaki et al. (6) that aortic constriction can still induce LVH in Agtr $1 A^{-/-}$mice and that $\mathrm{AT}_{1}$ is only partially responsible for $\mathrm{LVH}$. This latter conclusion is in contradiction to the current consensus that $\mathrm{AT}_{1}$ and $\mathrm{AT}_{2}$ have effects in antagonistic to growth regulation. However, the present in vivo studies suggest that
$\mathrm{AT}_{2}$ and $\mathrm{p} 70^{\mathrm{S} 6 \mathrm{k}}$ can be determinants in regulating $\mathrm{LVH}$, and they support the paradoxical phenotypic observation that $\mathrm{AT}_{2}$ is essential for $\mathrm{LVH}$ rather than for its inhibition in the heart. However, it is not clear how these mechanisms are related to several reported $\mathrm{AT}_{2}$ functions $(28,29)$.

In summary, we have found that in Agtr2-/Y mice, the left ventricle maintains normal contractile function but hypertrophic response to pressure overload is completely lost. The expression of ventricle-specific genes and marked suppression of $\mathrm{p}^{70^{\mathrm{S} 6 \mathrm{k}}}$, which regulates the DNA synthesis in Agtr2-/Y mice, may provide an explanation for their loss of LVH response. Since a wide variety of other growth-regulating components are expressed normally, the present results indicate specific roles of $\mathrm{p} 70^{56 \mathrm{k}}$ regulation in the Agtr2- $/ Y$ mouse heart and suggest that it is a major factor in mechanisms of cardiac hypertrophy.

\section{Acknowledgments}

This study was supported in part by NIH grants HL-58205 and DK-20593. We thank Mona Nemer for probes for Nkx2.5, GATA4, and MEF2C; Ronald Forczek and Peggy Petty for technical assistance with echocardiography; Erwin Landon for reading the manuscript; and Tina Stack for secretarial assistance.

1. Baker, K.M., and Aceto, J.F. 1990. Angiotensin II stimulation of protein synthesis and cell growth in chick heart cells. Am. J. Physiol. 259:H610-H618.

2. Sadoshima, J., Xu, Y., Slayter, H.S., and Izumo, S. 1993. Autocrine release of angiotensin II mediates stretch-induced hypertrophy of cardiac myocytes in vitro. Cell. 75:977-984.

3. Pfeffer, J.M., Pfeffer, M.A., Mirsky, I., and Braunwald, E. 1982. Regression of left ventricular hypertrophy and prevention of left ventricular dysfunction by captopril in the spontaneously hypertensive rat. Proc. Natl. Acad. Sci. USA. 79:3310-3314.

4. Lopez, J.J., et al. 1994. Distribution and function of cardiac angiotensin AT1- and AT2-receptor subtypes in hypertrophied rat hearts. Am. J. Physiol. 267:H844-H852.

5. Harada, K., et al. 1998. Pressure overload induces cardiac hypertrophy in angiotensin II type $1 \mathrm{~A}$ receptor knockout mice. Circulation. 97:1952-1959.

6. Hamawaki, M., et al. 1998. Pressure-overload hypertrophy is unabated in mice devoid of AT1A receptors. Am. J. Physiol. 274:H868-H873.

7. Ichiki, T., et al. 1995. Effects on blood pressure and exploratory behaviour of mice lacking angiotensin II type-2 receptor. Nature. 377:748-750.

8. Komuro, I., Kurabayashi, M., Takaku, F., and Yazaki, Y. 1988. Expression of cellular oncogenes in the myocardium during the developmental stage and pressure-overloaded hypertrophy of the rat heart. Circ. Res. 62:1075-1079.

9. Fentzke, R.C., et al. 1997. Evaluation of ventricular and arterial hemodynamics in anesthetized closed- chest mice. J. Am. Soc. Echocardiogr. 10:915-925.

10. Manning, W.J., Wei, J.Y., Katz, S.E., Litwin, S.E., and Douglas, P.S. 1994. In vivo assessment of LV mass in mice using high-frequency cardiac ultrasound: necropsy validation. Am. J. Physiol. 266:H1672-H1675.

11. Towbin, H., Staehelin, T., and Gordon, J. 1979. Electrophoretic transfer of proteins from polyacrylamide gels to nitrocellulose sheets: procedure and some applications. Proc. Natl. Acad. Sci. USA. 76:4350-4354.

12. Rockman, H.A., et al. 1991. Segregation of atrialspecific and inducible expression of an atrial natriuretic factor transgene in an in vivo murine model of cardiac hypertrophy. Proc. Natl. Acad. Sci. USA. 88:8277-8281.

13. Krieg, P.A., and Melton, D.A. 1987. In vitro RNA synthesis with SP6 RNA polymerase. Methods Enzymol. 155:397-415

14. Matsui, H., Pallen, C.J., Adachi, A.M., Wang, J.H., and Lam, P.H. 1985. Demonstration of different metal ion-induced calcineurin conformations using a monoclonal antibody. J. Biol. Chem. 260:4174-4179.

15. Sadoshima, J., and Izumo, S. 1995. Rapamycin selectively inhibits angiotensin II-induced increase in protein synthesis in cardiac myocytes in vitro. Potential role of $70-\mathrm{kD}$ S6 kinase in angiotensin II-induced cardiac hypertrophy. Circ. Res. 77:1040-1052.

16. Thorburn, J., Frost, J.A., and Thorburn, A. 1994. Mitogen-activated protein kinases mediate changes in gene expression, but not cytoskeletal organization associated with cardiac muscle cell hypertrophy. J. Cell Biol. 126:1565-1572.

17. Glennon, P.E., et al. 1996. Depletion of mitogenactivated protein kinase using an antisense oligodeoxynucleotide approach downregulates the phenylephrine-induced hypertrophic response in rat cardiac myocytes. Circ. Res. 78:954-961.

18. Kudoh, S., et al. 1997. Angiotensin II stimulates c-Jun NH2-terminal kinase in cultured cardiac myocytes of neonatal rats. Circ. Res. 80:139-146.

19. Hirota, H., et al. 1999. Loss of a gp130 cardiac muscle cell survival pathway is a critical event in the onset of heart failure during biomechanical stress. Cell. 97:189-198.

20. Matsubara, H. 1998. Pathophysiological role of angiotensin II type 2 receptor in cardiovascular and renal diseases. Circ. Res. 83:1182-1191.

21. Bartunek, J., et al. 2000. Chronic N(G)-nitro-Larginine methyl ester-induced hypertension: novel molecular adaptation to systolic load in absence of hypertrophy. Circulation. 101:423-429.

22. Levine, H.J. 1972. Compliance of the left ventricle. Circulation. 46:423-426.

23. Mukherjee, D., and Sen, S. 1991. Alteration of collagen phenotypes in ischemic cardiomyopathy. J. Clin. Invest. 88:1141-1146.

24. Schluter, K.D., and Piper, H.M. 1999. Regulation of growth in the adult cardiomyocytes. FASEB J. 13(Suppl.):S17-S22.

25. Yamazaki, T., et al. 1993. Mechanical loading activates mitogen-activated protein kinase and S6 peptide kinase in cultured rat cardiac myocytes. J. Biol. Chem. 268:12069-12076.

26. Arai, H., et al. 1988. Augmented expression of atrial natriuretic polypeptide gene in ventricles of spontaneously hypertensive rats (SHR) and SHRstroke prone. Circ. Res. 62:926-930.

27. Molkentin, J.D., et al. 1998. A calcineurindependent transcriptional pathway for cardiac hypertrophy. Cell. 93:215-228.

28. Lokuta, A.J., Cooper, C., Gaa, S.T., Wang, H.E., and Rogers, T.B. 1994. Angiotensin II stimulates the release of phospholipid-derived second messengers through multiple receptor subtypes in heart cells. J. Biol. Chem. 269:4832-4838.

29. Martens, J.R., Wang, D., Sumners, C., Posner, P. and Gelband, C.H. 1996. Angiotensin II type 2 receptor-mediated regulation of rat neuronal $\mathrm{K}^{+}$ channels. Circ. Res. 79:302-309. 